
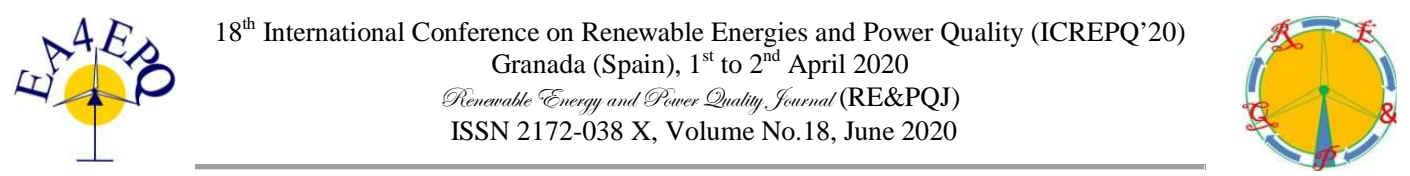

\title{
Assessment of the Royal Decree 244/2019 in the Spanish Electrical Regulatory Framework Considering Power Quality Issues related to Harmonic Distortion associated to Nonlinear loads in Grid-connected Microgrids
}

\author{
J. El Mariachet ${ }^{1}$, J. Matas ${ }^{1}$, H. Martin ${ }^{1},{ }^{\prime}$. Anzúrez ${ }^{2}$, G. Tinoco ${ }^{2}$, Israel Luna Reyes ${ }^{2}$,S. Abdalinejad ${ }^{1}$ \\ ${ }^{1}$ Department of Electrical Engineering \\ EEBE, Polytechnic University of Catalonia, Barcelona (Spain) \\ ${ }^{2}$ Department of Electrical Engineering \\ FIE, Universidad Michoacana de San Nicolás de Hidalgo, Morelia (Mexico)
}

Phone number:+0034 634535033, e-mail: jorge.el.mariachet@upc.edu

\begin{abstract}
Distributed Generation (DG) in Spain has recently been boosted through the impact in the regulatory framework of the electrical grid and the electrical market provided by the Royal Decree 244/2019. One important novelty is that it incorporates to the Royal Decree 842/2002 a modification establishing that prosumers associated to a self-consumption Grid-connected Microgrid (GCMG) classified as without energy surplus, cannot deliver energy to the electrical network. The criteria for ensuring this restriction only consider classical Power Factor (PF) calculation, Overvoltage and DC current injection and do not consider Power Quality (PQ) issues related to Total Harmonic Distortion (THD). In the recent years, nonlinear loads are increasing its presence in the electrical installations consumption side. Nonlinear loads such as lighting, electronic devices and others can pollute with harmonics the GCMG and, therefore, the Grid. This work proposes an assessment of the modifications of the Royal Decree 842/2002 considering PQ issues related to THD due to the Nonlinear Loads existing on a GCMG in the context of the Smart Grid (SG).
\end{abstract}

Key words. Real Decreto 244/2019, Energy Transition, Smart Metering, Power Quality, Subharmonics, Smart Grid.

\section{Introduction}

Energy Transition in Spain is being scheduled accordingly to [1]. An Integrated Energy and Climate Plan [2] has been implemented by the government authorities in order to fulfil with the requirements of the "2030 Climate and Energy Policy Framework" [3]. In this context the Royal Decree 244/2018 [4] provides a new regulation to the technical conditions for the prosumption of electrical energy that directly modifies the low voltage regulations for AC single-phase and three-phase electrical power systems [5]. Specifically, in the Final Disposition Nr. 2 of [4] it estates that any GCMG defined as a selfconsumption installation without surpluses in [4]-[6], must avoid the injection of energy to the Grid by means of compensation, storage or directly disconnection of the generation resources. The main condition is that the generated energy must be always lower than the consumed energy. The control is proposed to relay on a measurement equipment that belongs to each prosumer. Finally, the Transmission System Operators (TSO) and the Distribution System Operators (DSO) need to estimate the power losses on their Low Voltage (LV) lines. This is achieved through the collection and management of active and reactive power data, $P_{i}$ and $Q_{i}$ respectively, related to the Consumer Profile Load (CPL) automatically assigned to each prosumer by Smart Metering devices [10]. The estimation of these losses is then based only on the averaged values of $P_{i}$ and $Q_{i}$ that doesn't take into account THD pollution. On the other hand, accordingly to [4], a broad adoption of renewable energy technologies by the users can be expected in the following years, as suggested in [7]. Then PQ issues related to harmonic distortion, reliability, stability, among others, will arise. Nowadays, the technologies for ensuring a proper $\mathrm{PQ}$ in the Microgrids (MG) are in constant evolution, since [8]. Therefore, accordingly to Figure 1, the measurement equipment $M$ installed in GCMG can be upgraded for offline regulation with respect to the Grid. Even more, the calculation of the generated energy in the GCMG should be carried out in the same manner than the company equipment's Nevertheless the measurement equipment installed by the DTO or TSO cannot be as easily upgraded due to the requirements imposed by [9] to the Active Energy Meters, that only considers harmonic and DC as long duration disturbances, but not SSFP. This fact has a negative influence on the estimation of power losses in the distribution lines of DTO or TSO. The following Section 2 shows the limitations of the measurement equipment's based on [10]-[12] and considering [13]. In Section 3 simulations of MG with Linear and Nonlinear loads with low frequency perturbations in voltage are achieved and in Section IV a proposal of modification of [5] is presented accordingly to the simulation results. 


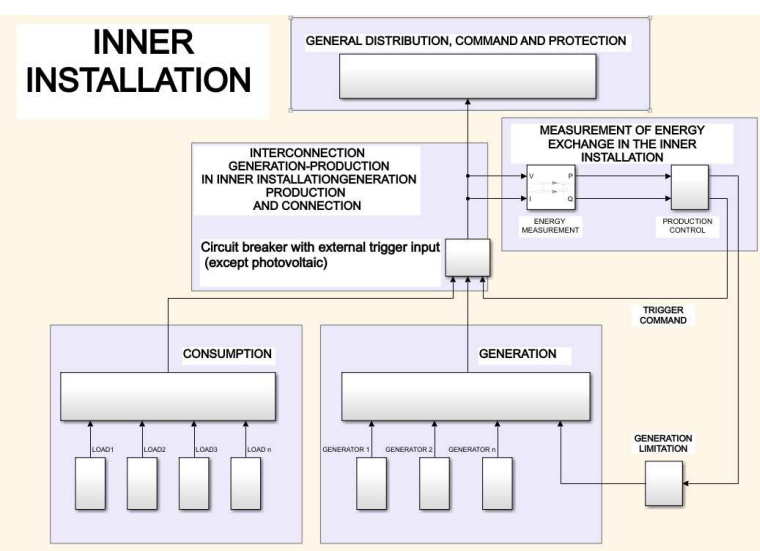

Fig. 1. Scheme of a GCMG for Self-consumption installation without surplus; Source: RD244/2019 [4]

\section{Smart Metering Power Calculation}

The smart Meters employed by the distribution companies are aimed to calculate the instantaneous active assuming that voltage and current are expressed as (1) and (2) respectively, from [8] or [9] :

$$
\begin{aligned}
& v(t)=V_{D C}+\sum_{n=1}^{N} V_{n} \cdot \sin \left(n \omega_{o} \cdot t+\alpha_{n}\right) \\
& i(t)=I_{D C}+\sum_{n=1}^{N} I_{n} \cdot \sin \left(n \omega_{o} \cdot t+\beta_{n}\right)
\end{aligned}
$$

where $n$ is the harmonic order, $\omega_{o}$ the fundamental frequency of the grid, $\alpha_{n}$ and $\beta_{n}$ the harmonic phase shift for voltage and current respectively, $V_{D C}$ and $I_{D C}$ the continuous componets for voltage and current and $\mathrm{N}$ is the maximum number of harmonics observed. Then, considering $\alpha_{n}=0$, a $90^{\circ}$ delay is applied to the voltage, for the obtention of the active and reactive instantaneous powers as indicates (3) and (4) respectively:

$$
\begin{aligned}
& p(t)=\sum_{n=1}^{N} v_{n}(t) \cdot i_{n}(t) \\
& q(t)=\sum_{n=1}^{N} v_{n \perp}(t) \cdot i_{n}(t)
\end{aligned}
$$

being $v_{n}(t)$ and $i_{n}(t)$ the harmonic components for voltage and current, $v_{n \perp}(t)$ the $90^{\circ}$ delayed voltage. This operation is repeated each $50 \mathrm{~ms}$ as (3) and (4) are averaged after a minute. Finally, However this assumption is not correct when $\alpha_{n} \neq 0$, subsynchronous frequency perturbations (SSFP) and Interharmonics are present [13]. The superposition principle implies that the distributive property must be applied in the product of (1) and (2), which leads to the expressions reported in [13] and [14]. Even more, if only SSFP are considered, there is a loss of information in (3) and (4) related to the energy contained in low frequencies. Therefore, although the measurement of energy in the GCMG can be accurate and the difference between the consumed and generated by the prosumer remains positive, undesired energy and perturbations are injected to the grid.

\section{Nonlinear Loads GCMG Simulations}

In [7] it is suggested that once the regulatory framework and the economic cost for prosumption are favourable enough, novel PQ issues will arise related to a wide use of MG in a DG based Smart Grid. In this section an ideal single-phase voltage source with a SSFP of a $5 \%$ in amplitude and different frequencies will be simulated supplying to a nonlinear load. The Nonlinear load is composed by a Diode Bridge connected to a RC branch. The parameters of the simulation are listed in Table I.

Table I. - Simulation Parameters

\begin{tabular}{|l|c|}
\hline \multicolumn{1}{|c|}{ Parameter } & Value \\
\hline R-C & $1100 \Omega-470 \mu$ \\
\hline Vo & $311 \mathrm{~V}$ \\
\hline VSSFP & $15.55 \mathrm{~V}$ \\
\hline$\omega_{\mathrm{o}}$ & $100 \pi \mathrm{rad}^{-1}$ \\
\hline$\omega_{\text {SSFP }}($ Figures $2,3,4)$ & $(50 \pi, 10 \pi, 10 \pi) \mathrm{rad}^{-1}$ \\
\hline
\end{tabular}

Table II. - Simulation Results

\begin{tabular}{|c|c|l|}
\hline Analysed parameter & $\omega_{\text {SSFP }=} \mathbf{5 0} \boldsymbol{\pi} \mathrm{rad}^{-1}$ & $\omega_{\text {SSFP }}=\mathbf{1 0} \boldsymbol{\pi}$ rad $^{-1}$ \\
\hline Output current not filtered & $\mathrm{THD}=225.6 \%$ & $\mathrm{THD}=231.91 \%$ \\
\hline Output current filtered & $\mathrm{THD}=5.58 \%$ & $\mathrm{THD}=5.46 \%$ \\
\hline \multicolumn{2}{|c|}{ ACTIVE POWER CALCULATION } \\
\hline \multicolumn{2}{|c|}{ Averaged Value } & THD (respect to DC) \\
\hline 247W & $5.12 \%$ \\
\hline
\end{tabular}

Figure 2 shows the distorted voltage waveform, the distorted current waveform with its FFT analysis. Figure 3 shows the same plots than figure 2 but adding a SSFP in voltage of $5 \mathrm{~Hz}$ instead of $25 \mathrm{~Hz}$. The SSFP amplitude has been kept constant at $5 \%$ of the fundamental component. The simulations had been carried out by means of Matlab/Simulink. As can be seen in Figure 2 and 3 , there is a region below the fundamental component for the nonlinear current that is not considered in (4). This is more consistent with the proposed expressions for the current in [13]. Figure 4 shows the filtered current resulting after the $5 \mathrm{~Hz}$ voltage SSFP component, that presents only a $\mathrm{THD}=5.46 \%$. For the calculation of the averaged active power the algorithm shown in [13] and [15] is employed.

A FFT analysis with respect the DC component is carried out for the active power. The Dc component is the calculated active power in the system. The resulting plots can be seen in Figure 7, that shows the ripple of the calculated active power in steady state and the spectra bar diagram with respect to DC. If the FFT lists shown in Figure 5 and 6 for the filtered voltage and current, respectively, are taken into account, there should only be a component of the active power at $\omega=50 \mathrm{~Hz}$, mainly due to (3) at $25 \mathrm{~Hz}$. However, there appear several components in the spectra of the power with respect to the DC component, signalled with the red arrows in Figure 7, that are more relevant than the double frequency component. Even more, the phase shift for the voltage SSFP component at $25 \mathrm{~Hz}$ in figure 5 is around $90^{\circ}$, which is almost in phase with the current SSFP component at $25 \mathrm{~Hz}$. 

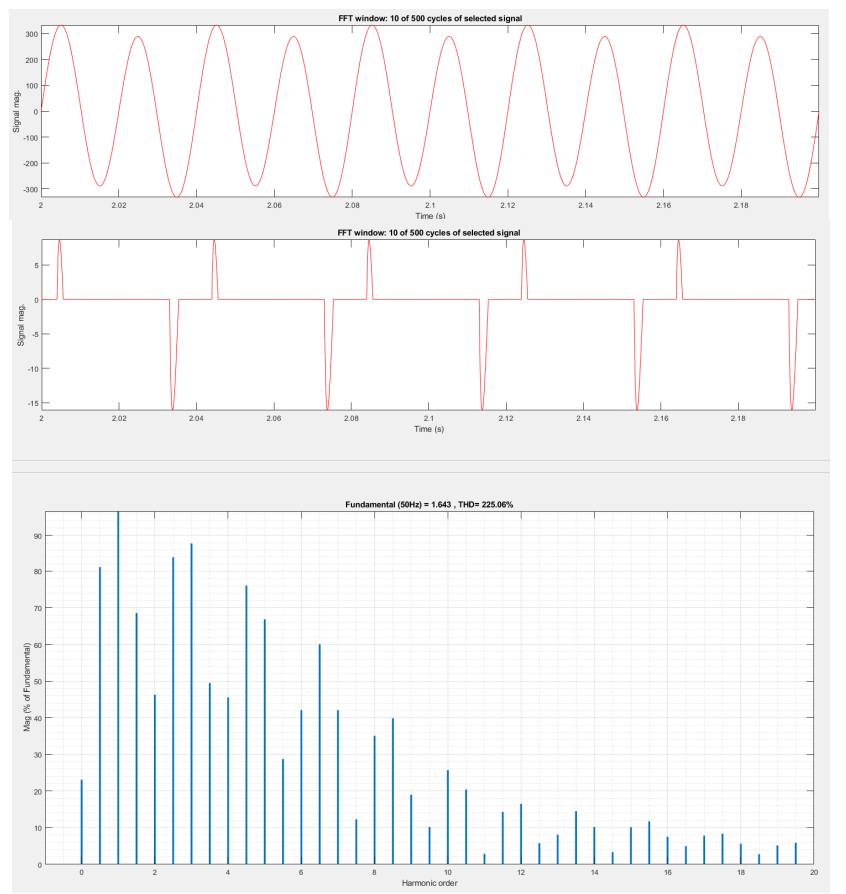

Figure 2: a) Non filtered Voltage signal with the 25Hz SSFP; b) Non filtered current measured in the Nonlinear load; c) FFT analysis of the current.

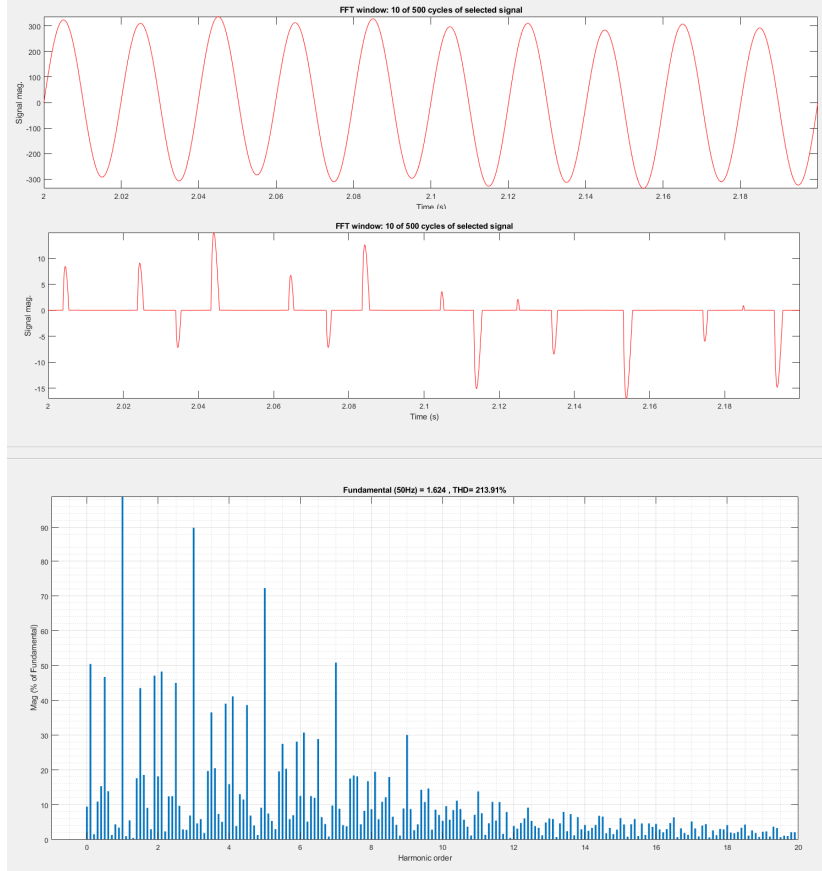

Figure 3: a) Non filtered Voltage signal with the 5Hz SSFP perturbation; b) Non filtered current signal measured in the Nonlinear load; c) FFT analysis of the current.

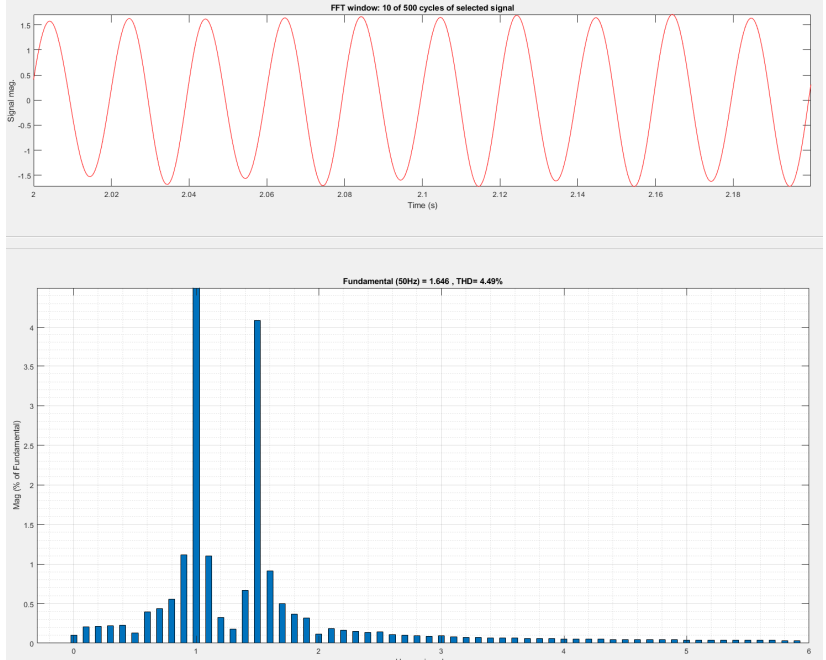

Figure 4: a) Filtered output current through the algorithm presented in [13] and [15]; b) FFT analysis of the filtered current.
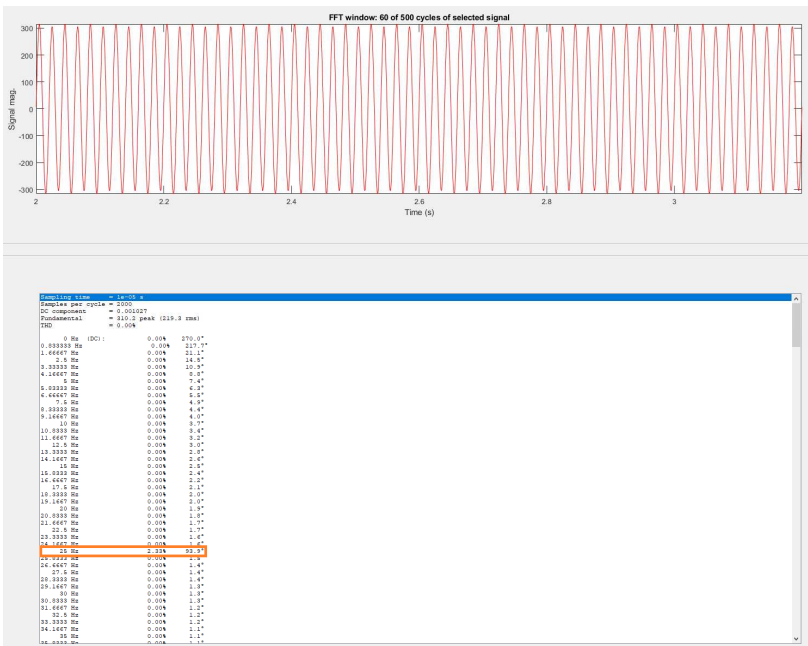

Figure 5: Filtered voltage and the FFT listing for magnitudes and phases for low frequency

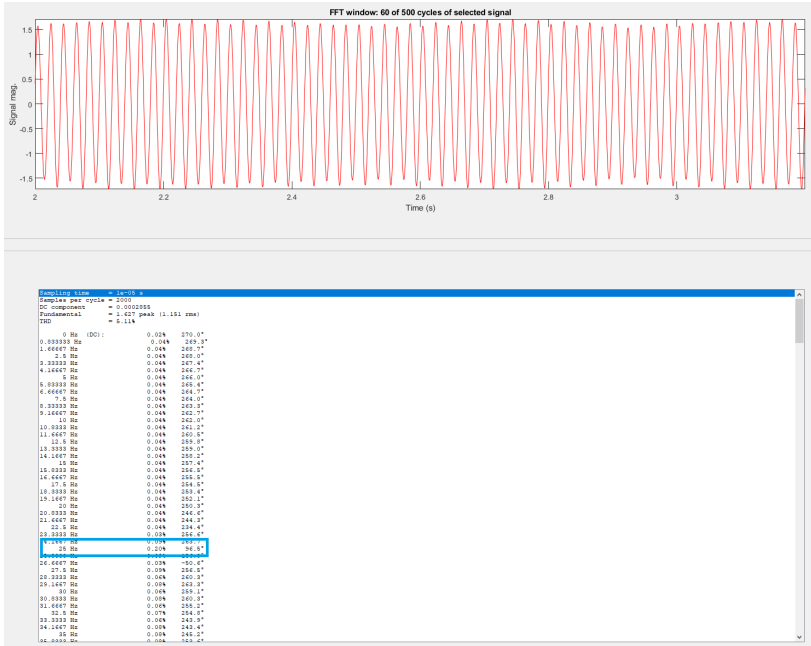

Figure 6: Filtered output current signal and listing for magnitudes and phases for low frequency 


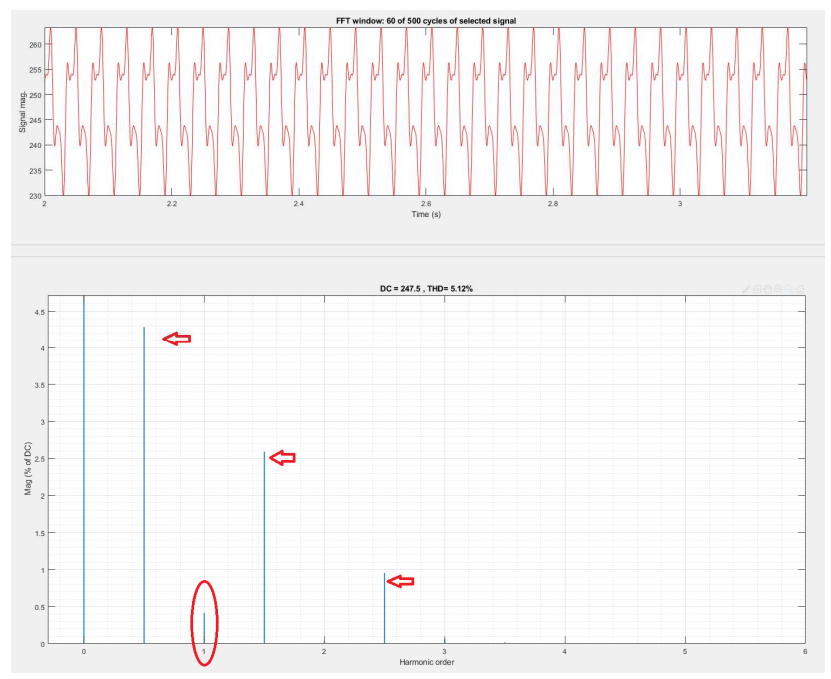

Figure 7: Averaged active power calculation after filtering the nonlinear current.

\section{Proposal of Modification of RD842/2002 and RD244/2018 considering SSFP.}

The amendment of the $40^{\text {th }}$ Complementary Technical Instruction for Low Voltage of [5], known by its ITC-BT40 Spanish acronym, should include in its Appendix I that the measurement device in Figure 1 must avoid the harmonic distortion due to SSFP, which are not DC current components. Regarding to the energy injection to the electrical grid network, Appendix I of [4] establishes a calculation of the energy per hour as follows:

$$
E N G_{h, i}=\beta_{i} \cdot E N G_{h}
$$

where $E N G_{h}$ is the generated energy by the GCMG and $\beta_{i}$ is defined as the proportional share of a prosumer associated to a common installation with other prosumers. If the prosumer $i$ installation is mainly composed by nonlinear loads as the described in this work, the parameter $\beta_{i}$ should be adapted at least as follows, for considering SSFP:

$$
\beta_{i}^{\prime}=\frac{\beta_{i}}{T H D_{S S F P}} 100
$$

and

$$
E N G_{h, i}=\beta_{i}^{\prime} \cdot E N G_{h}
$$

being THD $_{\text {SSFP }}$ the Total Harmonic Distortion due of the SSFP non-filtered of the currents of Table II. Note that for typical frequencies of $1 \mathrm{~Hz}$ or below the time window for calculating this SSFP THD should be larger than the $2 \mathrm{~s}$ left for detection in [4], as it was indicated in [15]. Then, the control algorithm for the scheme depicted in Figure 1 should take into account this THD $_{\text {SSFP }}$ parameter in order to avoid (8) in the case of self-consumption without surplus:

$$
\text { Eaut }_{h, i} \geq E N G_{h, i}
$$

where Eaut thi $_{\text {in }}$ is the consumed energy of a specific prosumer $i$. If the TSO and DTO need to properly estimate its losses following [10], that means that other prosumers sharing the same GCMG with linear loads should apply techniques for keeping (9), it is, to avoid to inject energy to the grid:

$$
V_{h, k}=\alpha_{k} \cdot\left(E N G_{h, k}-\sum_{i} E_{a u t_{h, i}}\right)=0
$$

where $k$ is the GCMG shared by $i$ prosumers, $V_{h, k}$ is the daily injected energy to the Grid, $\alpha_{k}$ the coefficient defined for the sharing of the daily generated energy of the installation $\mathrm{k}$, accordingly to [4].

\section{Conclusion}

Accordingly to [4], the electrical market in Spain will be influenced by novel PQ issues, regarding [8]. The increasing presence of electronic switching nonlinear loads as well as wind generation, among others, may introduce SSFP that can undermine the stated scheme in Figure 1 of [4]-[5] and the criteria for non-injection of energy to the Grid in certain cases determined in [4] and [6]. The injection to the DTO's LV grid network of energy associated to SSFP while the generators of the GCMG are connected may provoke errors in the estimation of the losses in the lines of the DTO and TSO related in [10]. The simulation results of this work point in the direction that the definitions for Active and Reactive power in [10]-[12] are not suitable for these losses estimations in the electrical grid, neither for controlling the connection-disconnection of the generation equipment in GCMG through the devices type $M$ depicted in Figure 1. Therefore, a novel parameter $\beta_{i}^{\prime}$

is proposed in order to better balance the sharing of the generated energy in a GCMG for being included in the RD244/2018 that modifies ITC-BT-40 of RD842/2002. Future work is directed to analyse how to improve the losses estimations for the power management in the Smart Grid considering novel PQ issues.

\section{References}

[1] COM/2015/80 final, of 25th February 2015, European Commission, "A Framework Strategy for a Resilient Energy Union with a Forward-Looking Climate Change Policy", Document 52015DC0080 .https://eur-lex.europa.eu/legalcontent/EN/TXT/?uri=CELEX:52015DC0080. Last access online September 2019.

[2] Ministry for the Ecological Transition, February 2019, "Draft of the Integrated Energy and Climate Plan 2021-2030". https://ec.europa.eu/energy/sites/ener/files/documents/spain_draftn ecp.pdf. Last access online August 2019.

[3] COM/2014/15 final, of 22th April 2014, European Commission, "A policy framework for climate and energy in the period from 2020 to 2030", Document 52014DC0015. https://eurlex.europa.eu/legal-content/EN/TXT/?uri=CELEX:52014DC0015. Last access online September 2019.

[4] Royal Decree 244/2019, of 5th April. BOE no. 83, 6th April, 2019. https://www.boe.es/eli/es/rd/2019/04/05/244, pp. 3567435719. Last access online, October 2019.

[5] Royal decree 842/2002, of 2nd August. BOE no. 224, 18th September, 2019. https://www.boe.es/eli/es/rd/2002/08/02/842, pp. 33084-33086. Last access online, October 2019.

[6] Law 24/2013, of 26th December. BOE no. 310, $27^{\text {th }}$ December, 2013. https://www.boe.es/eli/es/1/2013/12/26/24. Last access online, October 2019.

[7] Hirsch, Adam \& Parag, Yael \& Guerrero, Josep, 2018 "Microgrids: A review of technologies, key drivers, and outstanding issues,"Renewable and Sustainable Energy Reviews, Elsevier, vol. 90(C), pages 402-411

[8] Naderi, Yahya \& Hosseini, Seyed Hossein \& Ghassem Zadeh, Saeid \& Mohammadi-Ivatloo, Behnam \& Vasquez, Juan C. \& Guerrero, Josep M., 2018. "An overview of power quality enhancement techniques applied to distributed generation in 
electrical distribution networks," Renewable and Sustainable Energy Reviews, Elsevier, vol. 93(C), pages 201-214

[9] European Parliament, OJL 135, 30.4.2004, "Directive 2004/22/EC, of 31 March 2004 on measuring instruments". https://eurlex.europa.eu/legal-content/EN/ALL/?uri=CELEX:32004L0022. Last access online August 2019.

[10] G. Grigoras and M. Gavrilas, "An Improved Approach for Energy Losses Calculation in Low Voltage Distribution Networks based on the Smart Meter Data," 2018 International Conference and Exposition on Electrical And Power Engineering (EPE), Iasi, 2018, pp. 0749-0754.

[11] IEEE Standard Definitions for the Measurement of Electric Power Quantities Under Sinusoidal, Nonsinusoidal, Balanced, or Unbalanced Conditions - Redline," in IEEE Std 1459-2010 (Revision of IEEE Std 1459-2000) - Redline, vol., no., pp.1-52, March 192010.

[12] IEC 61000 4-7, Electromagnetic compatibility (EMC) - Part 4-7: Testing and measurement techniques - General guide on harmonics and interharmonics measurements and instrumentation, for power supply systems and equipment connected thereto

[13] J. El Mariachet, J. Matas, Helena Martín, Abdullah Abusorrah, "Power Calculation Algorithm for Single-Phase Droop-Operated Inverters Considering Nonlinear Loads," in 2018 International Conference on Renewable Energies and Power Quality (ICREPQ), Salamanca, April 2018, pp.19-25.

[14] Hyosung Kim, F. Blaabjerg and B. Bak-Jensen, "Spectral analysis of instantaneous powers in single-phase and three-phase systems with use of p-q-r theory," in IEEE Transactions on Power Electronics, vol. 17, no. 5, pp. 711-720, Sept. 2002.

[15] J. El Mariachet, J. Matas, H. Martín, G. Tinoco, S. Abdalinejad, "Proposal of Novel Single-Phase Power Quality Indicators considering subsynchronous frequency perturbations in Voltage and Current under non-sinusoidal conditions", in 2019 International Conference on Renewable Energies and Power Quality (ICREPQ), La Laguna, April 2019, pp.530-535 